\title{
ANÁLISE DA VARIABILIDADE ESPACIAL DA RESISTÊNCIA À PENETRAÇÃO DO SOLO EM DIFERENTES PROFUNDIDADES
}

${ }^{1}$ UNESP - Universidade Estadual Paulista, Faculdade de Ciências Agronômicas de Botucatu, Campus de Botucatu, SP, Brasil.

${ }^{2}$ UNESP - Universidade Estadual Paulista, Faculdade de Ciências e Engenharia, Campus de Tupã, SP, Brasil.

${ }^{3}$ Fatec - Faculdade de Tecnologia, Shunji Nishimura, Campus de Pompéia - SP, Brasil.

Article history: Received 11 April 2018; Received in revised form 10 June 2018; Accepted 13 June 2018; Available online 28 June 2018.

\section{RESUMO}

A compactação do solo em áreas cultivada cana-de-açúcar é muito acentuada devido ao uso intenso de máquinas e implementos agrícolas, é um dos fatores que ocasionam sérias restrições ao desenvolvimento radicular das plantas. Objetivou-se neste trabalho caracterizar a variabilidade espacial da resistência do solo a penetração (RSP), de uma área reformada após acomodar por cinco anos plantio de cana-de-açúcar. O experimento foi conduzido em abril de 2015 na Fazenda Santa Martha localizada em Espírito Santo do Turvo, SP. A coleta da RSP foi feita por meio de um penetrômetro elétrico, em pontos previamente georreferenciados totalizando 56 pontos, distribuídos em um talhão irregular. Utilizou-se a geoestatística para análise das variações espaciais da RSP de cinco diferentes profundidades do perfil do solo (1, 10, 20, 30 e $40 \mathrm{~cm}$ ), foi feita também a coleta da umidade do solo, somente nos pontos centrais. O modelo de semivariograma que melhor se ajustou aos valores de resistência do solo à penetração foi o esférico. Este estudo resultou em altos e moderados graus de dependência espacial, nos perfis de 1,10 e $20 \mathrm{~cm}$, e efeito pepita nas profundidades 30 e 40 $\mathrm{cm}$. Foi possível avaliar a variabilidade espacial da RSP por meio da geoestatística, sendo que a maior homogeneidade foi no perfil de 1,10 e $20 \mathrm{~cm}$.

Palavras-chave: Compactação do solo; geoestatística; Krigagem.

\section{ANALYSIS OF THE SPATIAL VARIABILITY OF RESISTANCE TO SOIL PENETRATION AT DIFFERENT DEPTHS}

\begin{abstract}
The compaction of the soil in sugarcane cultivated areas is very accentuated due to the intense use of machines and agricultural implements, it is one of the factors that cause serious restrictions to the root development of the plants. The objective of this work was to characterize the spatial variability of soil penetration resistance (SPR) of a reforested area after five years of sugar cane cultivation. The experiment was conducted in April 2015 at the Santa Martha Farm located in Espirito Santo do Turvo, SP. The SPR was collected by means of an electric penetrometer, in previously georeferenced points totaling 56 points, distributed in an irregular area. Geostatistics was used to analyze the spatial variations of SPR from five different soil depths $(1,10,20,30$ and $40 \mathrm{~cm})$. Soil moisture was collected only at central
\end{abstract}


points. The semivariogram model that best fit the values of SPR was spherical. This study resulted in high and moderate degrees of spatial dependence, in the profiles of 1, 10 and 20 $\mathrm{cm}$, and pips effect in depths 30 and $40 \mathrm{~cm}$. It was possible to evaluate the spatial variability of RSP by means of geostatistics, with the highest homogeneity in the 1,10 and $20 \mathrm{~cm}$ profile.

Keywords: Soil compaction; geostatistics; kriging.

\section{INTRODUÇÃO}

O uso de maquinas e implementos agrícolas influencia diretamente na compactação do solo, sendo este um dos fatores que causam severas restrições ao crescimento radicular das plantas (ROQUE et al., 2010; LIMA et al., 2013). O sistema radicular da cana-de-açúcar tende a se desenvolver com maior profundidade, quando confrontado com outras culturas, desenvolvida por rizomas e raízes fasciculadas, das quais $60 \%$ na camada de 20 a $30 \mathrm{~cm}$ de profundidade e $85 \%$ se encontram na camada de $50 \mathrm{~cm}$, explicando, portanto, o fato de ser uma das culturas mais afetadas pela compactação do solo (LIMA et al., 2013; OLIVEIRA et al., 2013).

A compactação do solo pode ser definida como a diminuição do volume de solo, ocasionada pela compressão do mesmo, onde ocorre uma reorganização densa das partículas constituintes (CURI et al., 1993). Os mesmos autores acrescentam, que este fenômeno se trata da redução natural do espaço poroso com consequente aumento da densidade de camadas por dessecação, iluviação ou precipitação química, dinâmica resultante da ação antrópica, ao passo que o adensamento é fenômeno natural.

Uma das mais relevantes e principais causas da deterioração do solo é a compactação (RALISCH et al., 2008). A textura do solo é um dos fatores importantes no processo de compactação, segundo Bacchi (1976), os solos mais susceptíveis são os de textura média à moderadamente grosseira (francos, franco arenosos e franco siltosos), devido a maior facilidade de reorganização de suas partículas. Os solos altamente porosos são mais passíveis ao processo de compactação que solos menos porosos.

A resistência do solo a penetração (RSP) é um dos parâmetros mais utilizados para avaliar o teor da compactação, pertinente aos atributos físicos do solo, considerado um indicador do grau de compactação, como a textura e umidade que influenciam diretamente desempenho do solo, submetido a pressões externas e sendo útil para localizar camadas de solo compactadas (ROQUE et al., 2008; COLET et al., 2009).

A RSP, por meio de penetrômetro, quantifica a força exercida no solo em função da penetração de uma ponta cônica, simulando a resistência que o solo oferece à penetração das raízes (MOLIN et al., 2012; LIMA et al., 2013).

Entre os cuidados para atenuar o problema de compactação, recomenda-se o preparo menos intenso possível do solo, tráfego leve, evitar trabalhar sobre o solo úmido, reduzir a pressão de contato das máquinas sobre o solo e realizar um planejamento adequado de safra. Outro manejo importante a se fazer em uma área é considerar a variabilidade espacial da RSP, conhecendo a distribuição heterogênea existente em uma área de modo a maneja-la de forma mais consciente. Para isso é necessário fazer um estudo da distribuição espacial por meio da geoestatística que possibilita estruturar a variabilidade natural dos atributos avaliados, principalmente quando a dependência espacial está dentro do intervalo da amostragem (MACHADO et al., 2007).

O uso da Krigagem ordinária como método de interpolação dos atributos do solo, possibilita o estudo da variabilidade 
espacial das propriedades do solo (BROCCA et al., 2007; OHASHI \& GYOKUSEN, 2007; ZHU \& SHAO, 2008; PANOSSO et al, 2009; BRITO et al.,2010).

Este estudo possibilita a interpretação dos resultados com base na estrutura da variabilidade natural dos atributos avaliados, considerando a dependência espacial dentro do intervalo de amostragem. Sabe-se que as variabilidades espaciais da resistência do

\section{MATERIAL E MÉTODOS}

O experimento foi realizado em abril de 2015, na Fazenda Santa Martha, localizada no município de Espírito Santo do Turvo, SP, (Latitude 22\%41'32" S, longitude $49^{\circ} 25^{\prime} 49^{\prime \prime} \mathrm{O}$, com altitude 520 metros). A fazenda ocupa uma área de solo à penetração não ocorrem ao acaso, mas apresentam correlação ou dependência espacial (CAMBARDELLA et al., 1994; VIEIRA, 2000; SILVA et al., 2004; SOUZA et al., 2006).

Este trabalho visa determinar a variabilidade da resistência a penetração do solo, caracterizado: Latossolo Vermelho Distrófico (EMBRAPA, 2006), nas profundidades $1,10,20,30$ e $40 \mathrm{~cm}$, posteriormente caracteriza-lo de acordo com o grau de dependência observado.

411,40 hectares, sendo a dimensão da parcela em estudo de 18,2 hectares, como ilustrado na Figura 1. O solo da área é classificado como Latossolo Vermelho Distrófico (EMBRAPA, 2006).

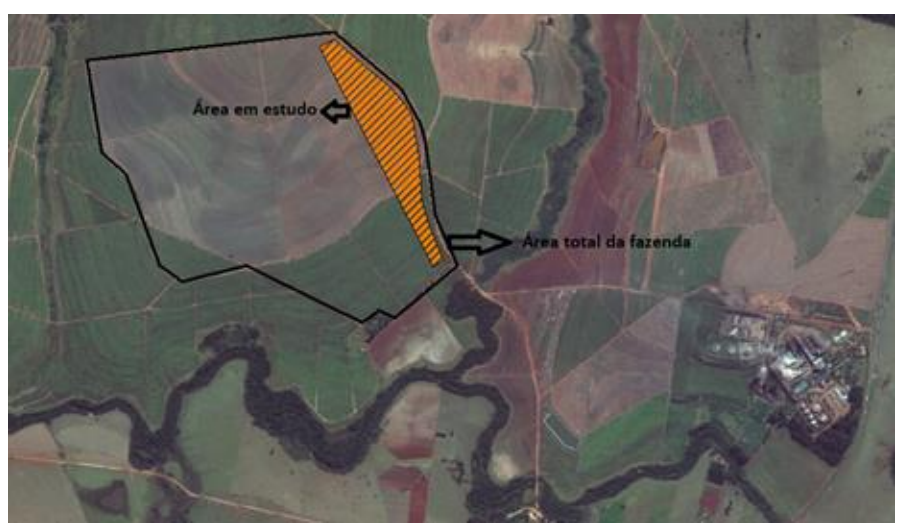

Figura 1. Área total da fazenda e em evidencia a área em estudo, adaptado de Google Earth

A cidade é caracterizada pelo clima subtropical úmido conforme classificação climática de Köppen: Aw (CEPAGRI, 2017), além disso, apresenta um verão úmido e inverno seco. Segue detalhado na figura 2, os fatores climáticos médios referentes a precipitação e temperaturas máximas e mínimas do município em estudo. 


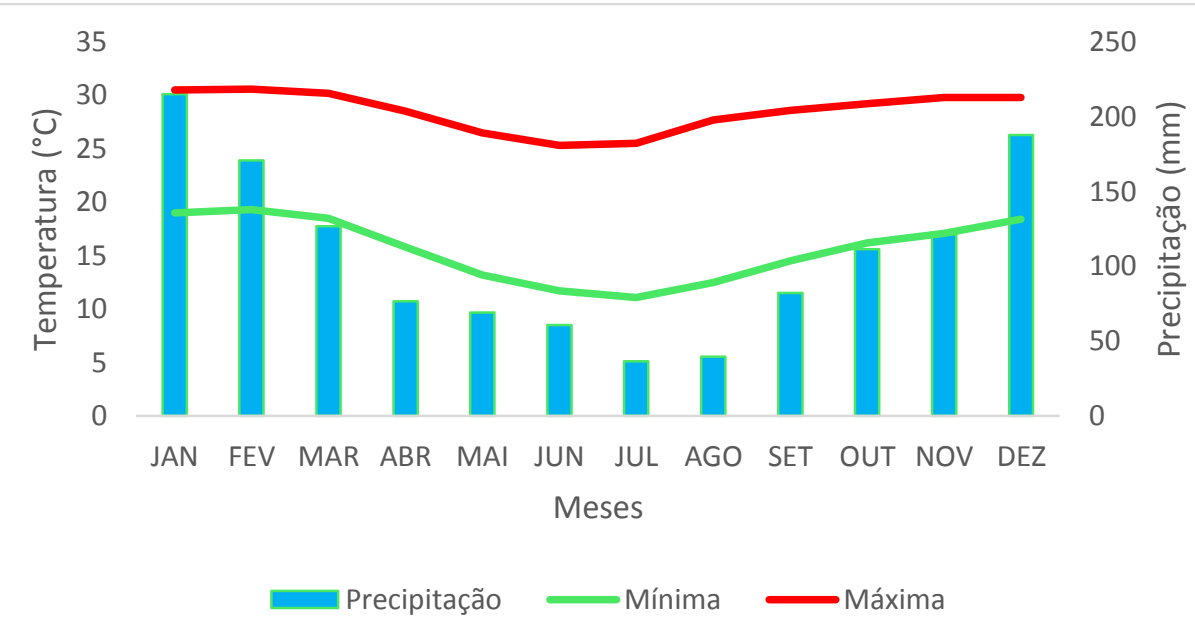

Figura 2. Precipitação e temperaturas mínimas e máximas anual da cidade Espírito Santo do Turvo. (CEPAGRI, 2017).

\section{Coleta de dados}

A área em estudo, acomodou um canavieiro durante 5 anos, cujo plantio foi feito por sulcação e a colheita foi totalmente mecanizada durante todo $\mathrm{o}$ período acomodado, esta técnica de colheita, submete o solo a pisoteio com máquinas pesadas, o qual pressiona o solo, deixando-o mais suscetível a altos teores de compactação.

Após retirada da cultura, a área foi submetida a reforma, como método de descompactação do solo, utilizou-se a grade aradora, que executou a quebra de partículas do solo à $25 \mathrm{~cm}$ de profundidade.

Os dados amostrais foram posicionados com espaçamento irregular, justificado pelo desenho do talhão, no entanto, o grid de base para posicionamentos dos pontos foi confeccionado com área de 100 x 100 metros, totalizando 56 pontos amostrais, seguindo $\mathrm{o}$ formato da área estudada georreferenciada, conforme Figura 3, em coordenadas UTM, Datum Horizontal WGS 84, Zona 22 Sul.

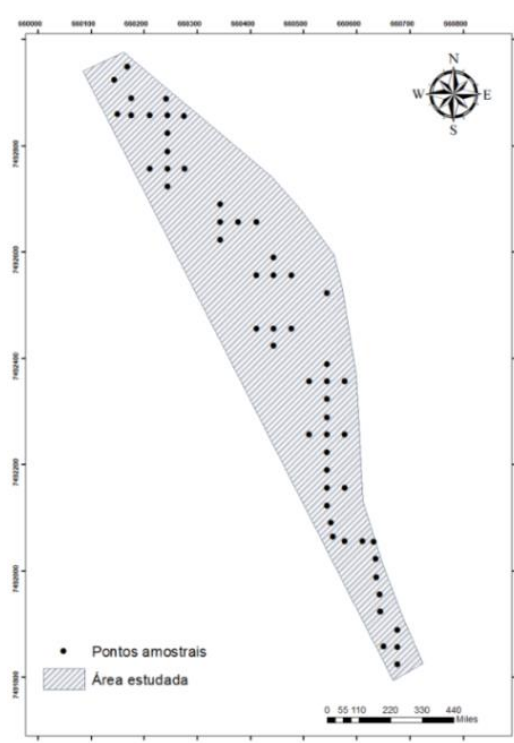

Figura 3. Grid da área estudada.

A demarcação dos pontos para coleta dos dados, foi obtida por meio do receptor GNSS de navegação (Sistema
Global de Navegação por Satélite), marca Garmim com precisão média de 5 metros. 
A compactação do solo foi determinada através de um penetrômetro Solo Track da marca Falker, elétrico e portátil, dotado de um sistema automático de medição de RSP. A variável determinada foi a RSP, ao longo do perfil do solo, 0-40 cm, em que foram estudadas as profundidades $1,10,20,30 \mathrm{e} 40 \mathrm{~cm}$, a força necessária para penetrar o solo foi quantificada em unidades mega pascal (MPa).

\section{Umidade do solo}

A coleta para obtenção do teor de água no solo, foi realizada em conjunto com as leituras de RSP. Este teor de água ou umidade do solo é determinado pela quantidade de água contida em uma amostra. $\mathrm{O}$ método para determinação da

$$
U_{s}=\frac{P_{u}-P_{s}}{P_{S}} \times 100
$$

em que:

$U_{s}$ : Umidade do solo (\%);

$P_{u}$ : Peso do solo úmido $(\mathrm{g})$;

\section{Estatística descritiva}

A caracterização inicial dos dados de $\mathrm{RSP}$, nas diferentes profundidades $(1,10$, 20,30 e $40 \mathrm{~cm}$ ) foi feito por meio de estatística descritiva, onde foram calculados os seguintes parâmetros: média aritmética, mediana, valores máximo e mínimos, desvio padrão, coeficiente de variação $(\mathrm{CV})$, o qual foi classificado segundo o método de Warrick (1998), baixo menor que $15 \%$, média quando está entre 15 e $50 \%$ e alto quando for maior que $50 \%$. Por fim, calculou-se os índices de assimetria e curtose.

umidade do solo foi o de estufa, conhecido também como método padrão.

As amostras foram coletadas em todos os pontos centrais do talhão, retiradas com ajuda de um trado, em seguida peneiradas para retirada de possíveis impurezas presentes no solo (objetos, pedras, vegetação e insetos). Posteriormente as amostras foram levadas para laboratório e acondicionadas em capsulas de alumínio devidamente identificadas e pesadas, sistemicamente com 100 gramas cada amostra de solo. Todas as amostras foram inseridas na estufa a $105^{\circ} \mathrm{C}$, permanecendo por 24 horas, após este período as amostras foram pesadas novamente e então encontrado o teor de água no solo por meio da Equação 1.

$$
\text { Eq. (1) }
$$

$P_{s}:$ Peso do solo seco $(\mathrm{g})$.

\section{Estatística Espacial (geoestatística)}

Para análise dos dados utilizou-se a geoestatística, associando o comportamento dos dados de RSP em diferentes profundidades no perfil do solo $(1,10,20,30$ e $40 \mathrm{~cm})$, avaliando todos os 56 pontos coletados.

A análise geoestatística é realizada, para avaliar a variabilidade dos dados analisados, com a verificação do grau de dependência espacial entre as observações, de acordo com a suposição de estacionariedade da hipótese intrínseca (VIEIRA \& COL, 1983), onde é estimada por meio da Equação 2:

$$
\gamma *(h)=\frac{1}{2 N(h)} \sum\left[\left(Z\left(x_{i}\right)-Z\left(x_{i}+h\right)\right]^{2}\right.
$$

Em que:

$\gamma *(h)$ : semivariância para um vetor $\mathrm{h}$; $Z(x) e Z(x+h)$ : são pares de observações;

$\mathrm{N}(\mathrm{h})$ : número de pares de valores medidos; $h$ : distância entre os pares de observações $[\mathrm{x},(\mathrm{x}+\mathrm{h})]$.

Os valores resultantes de $\gamma^{*}(h)$ versus $h$, é chamado de semivariograma experimental, capaz de expressar a variabilidade espacial entre as amostras, 
sendo uma função que só depende do vetor $\mathrm{h}$, portanto, dependem de ambos, magnitude e direção de $\mathrm{h}$. $\mathrm{O}$ ajuste do modelo matemático resultantes de $\gamma^{*}(h)$, são estimados através dos coeficientes: efeito pepita (Co), ideal que seja igual a 0 ; alcance (a), distância na qual as amostras apresentam-se correlacionadas espacialmente; patamar $(\mathrm{Co}+\mathrm{C} 1)$, valor do semivariograma correspondente a seu alcance (a).

Os semivariogramas foram obtidos por meio da variância dos dados, como orienta Vieira (1983). Os modelos geoestatísticos foram ajustados utilizando o software GS+7.0 (ROBERTSON, 2004), em que foi considerado a menor soma dos quadrados dos resíduos, o coeficiente de determinação R2 e coeficiente de correlação obtido pelo método de validação cruzada.

Os possíveis modelos de semivariograma a serem ajustados, através dos dados são: Esférico, $\gamma(\mathrm{h})=\mathrm{C} 0+\mathrm{C} 1\left[1,5(\mathrm{~h} / \mathrm{a})-0,5(\mathrm{~h} / \mathrm{a})^{3}\right]$ para $0<\mathrm{h}$

$$
G D E=\frac{C_{0}}{C_{0}+C} * 100
$$

Em que;

$G D E$ : Grau de dependência espacial;

$C_{0}$ : Efeito pepita;

$C_{0}+C$ : Patamar.

A classificação da dependência espacial dos dados são: para valores $\leq 25 \%$ é considerada forte; entre $25 \%$ e $75 \%$; moderada e $\geq 75 \%$ fraca.
$<$ a $\mathrm{e} \gamma(\mathrm{h})=\mathrm{C} 0+\mathrm{C} 1$ para $\mathrm{h} \geq \mathrm{a}$; Exponencial, $\gamma(\mathrm{h})=\mathrm{C} 0+\mathrm{C} 1[1-\exp (-$ 3h/a)] para $0<\mathrm{h}<\mathrm{d}$, em que d é a distância máxima na qual o semivariograma é definido; Gaussiano $\gamma(h)=C 0+C 1\left[1-\exp \left(-3 h^{2} / a^{2}\right)\right]$ para $0<h$ $<$ d.

A adequação do semivariograma através do ajuste do modelo matemático de cada profundidade estudada, disponibilizou os parâmetros: Efeito pepita $\left(C_{0}\right)$; Alcance (A) e Patamar $\left(C_{0}+C\right)$. Com isto, realizouse o calculo do grau de dependência espacial (GDE), o qual é caracterizado por indicar a confiança nas estimativas dos dados, por meio da variação espacial dos dados em relação à variância total (VIERA, 2000). Este índice foi comparado de duas formas Cambardella et al (1994) e Seidel \& Oliveira (2014).

A aquisição do GDE, segundo metodologia de Cambardela et al. (1994), consiste na relação entre patamar e efeito pepita, por meio da Equação 3.

Eq. (3)

A mensuração do GDE, pelo método de Seidel \& Oliveira (2014), considera todos os aspectos do semivariograma e por consequência características dados analisados, sendo eles, aspectos geométricos do modelo (Esférico, Exponencial e Gaussiano), distância máxima entre pontos e alcance prático, dada pela Equação 4.

$$
I D E=F M *\left(\frac{C_{0}}{C_{0}+C}\right) *\left(\frac{a}{q * M D}\right) * 100
$$

Em que:

$a$ : Alcance (m);

Segundo classificação dos autores do método, o GDE considera seus índices conforme ajuste do modelo dos semivarigramas, seguindo-se: entre $0 \mathrm{e}$ $50,4 \%$, para modelo gaussiano; entre 0 e $37,5 \%$, para modelo esférico e entre 0 e $31,7 \%$ para modelo exponencial.
$M D$ : Máxima distância entre pontos amostrados;

$q$ : fração máxima atingida da MD.

Após validação da dependência espacial dos dados, procedeu-se a interpolação dos mesmos para estimativa da RSP nos pontos não amostrados ao longo da extensão da área, sendo somente dados que apresentaram dependência espacial pelo método da Krigagem 
ordinária e aos que não atingiram dependência espacial, ou seja, efeito pepita

\section{RESULTADOS E DISCUSSÃO}

O ajuste dos semivariogramas modela e ilustra com maior acurácia o comportamento dos dados estudados. Segundo Ramos et al. (2007), em casos em que a distribuição não seja normal, mas razoavelmente simétrica, pode-se realizar a

\section{Estatística descritiva}

Analisando a estatística descritiva (Tabela 1) dos dados, observa-se que as amostras apresentaram médias crescentes, conforme aumento da profundidade $(1,10$, 20, 30 e $40 \mathrm{~cm}$ ), variando de 0.212 a 2.862 $\mathrm{MPa}$. Estudo semelhante foi realizado por Pereira et al, (2002), onde constatou-se essa mesma variação no estudo de comparação de diferentes níveis de compactação em ambiente de solo arenoso.

A diferença entre: média aritmética e mediana, apresentam valores bem puro, foram interpolados pelo método inverso da distância ponderada (IDW). construção do semivariograma. Os cálculos utilizados para análise geoestatística não necessitam conhecer a distribuição de frequências das variáveis analisadas (VIEIRA \& LOMBARDI NETO, 1995).

semelhantes, os quais estão diretamente ligados à distribuição normal dos dados. A normalidade ou não normalidade na distribuição dos dados, pode ocorrer por diversas causas, as quais pode ser por práticas de manejo ou efeitos temporais (CAMBARDELLA et. al, 1994).

$\mathrm{O}$ ajuste da frequência normal dos dados, não influencia na estatística espacial ela se baseia por meio de uma função aleatória contínua (VIEIRA 2000; ARZENO et. al, 2008).

Tabela 1: Estatística descritiva da RSP no perfil do solo.

\begin{tabular}{cccccccccc}
\hline Perfil & n & Média & Mínima & Máxima & Mediana & S & CV & Ck & Cs \\
\hline $1 \mathrm{~cm}$ & 56 & 0.212 & 0.090 & 0.540 & 0.160 & 0.1205 & 0.015 & -0.162 & 0.996 \\
$10 \mathrm{~cm}$ & 56 & 0.666 & 0.240 & 1.290 & 0.645 & 0.2449 & 0.060 & -0.481 & 0.439 \\
$20 \mathrm{~cm}$ & 56 & 1.452 & 0.550 & 2.760 & 1.445 & 0.7451 & 0.302 & -0.509 & 0.395 \\
$30 \mathrm{~cm}$ & 56 & 2.448 & 1.020 & 4.490 & 2.425 & 0.7451 & 0.555 & 0.439 & 0.537 \\
$40 \mathrm{~cm}$ & 56 & 2.862 & 1.260 & 4.780 & 2.860 & 0.7159 & 0.513 & 0.469 & 0.200 \\
\hline
\end{tabular}

Perfil: Amostras de RSP nas profundidades 1, 10, 20,30 e 40cm; n: número de amostras; s: desvio padrão; CV: coeficiente de variação; Ck: coeficiente de curtose; Cs: Coeficiente de assimetria.

\section{Estatística espacial (geoestatística)}

De acordo com os coeficientes de variação (CV), classificado por Warrick (1998), nas profundidades 1 e $10 \mathrm{~cm}$, foi considerado como baixo, provavelmente pelo efeito do manejo da descompactação do solo, já na profundidade $20 \mathrm{~cm}$, foi classificada como médio e por fim nas profundidades de 30 e $40 \mathrm{~cm}$, classificouse como baixa, indicando maior uniformidade da RSP nesta profundidade, estes resultados podem justificar maior uniformidade da compactação do solo. Quando os atributos apresentam alta variabilidade entre eles, as suas respectivas médias aritméticas podem não ser representativas em toda a área (CAMARGO et al., 2010). O qual também justifica este tipo de estudo.

A análise espacial é uma ferramenta importante para associar valores com sua localização no espaço e tempo, e colabora com o planejamento de ações de manejo relativas a resistência do solo à penetração (RSP), já que a distribuição contínua dos valores amostrados, permite a estimativa em locais não amostrados (LIMA et al., 2013).

A partir da Tabela 2, é possível analisar os ajustes dos modelos do 
semivariograma e suas respectivas variáveis, onde é possível notar que a predominância dos modelos ajustados foi o esférico, para as profundidades 1,10 e 20 e para as profundidades de 30 e $40 \mathrm{~cm}$, aconteceu efeito pepita puro (EPP). Segundo Campos et al. (2013) o modelo esférico e exponencial são os mais frequentes nas análises de atributos do solo.

Tabela 2: Parâmetros dos semivariogramas escalonados da RSP.

\begin{tabular}{ccccccccc}
\hline Atributos & $\begin{array}{c}\text { Modelo do } \\
\text { Semivariogra }\end{array}$ & Co & Co+C & A & R2 & GDE & MD & IDE \\
\hline $1 \mathrm{~cm}$ & Esférico & 0.005 & 0.024 & 958 & 0.94 & 20,83 & 616,73 & 29,62 \\
$10 \mathrm{~cm}$ & Esférico & 0.026 & 0.059 & 132 & 0.33 & 44,07 & 616,73 & 4,95 \\
$20 \mathrm{~cm}$ & Esférico & 0.176 & 0.354 & 301 & 0.58 & 49,72 & 616,73 & 9,18 \\
$30 \mathrm{~cm}$ & EPP & - & - & - & - & - & - & - \\
$40 \mathrm{~cm}$ & EPP & - & - & - & - & - & - & - \\
Umidade & EPP & - & - & - & - & - & - & - \\
\hline
\end{tabular}

Perfil: Amostras de RSP nas profundidades 1, 10, 20, 30 e 40cm; Co: Efeito pepita; Co+C: Patamar; A: Alcance; R2: coeficiente de determinação múltipla do ajuste; GDE: Grau de dependência espacial (método de Cambardella et al. (1994)); MD: Fração da máxima distância entre amostras; IDE: Índice de dependência espacial (Seidel \& Oliveira (2014).

Os alcances (A) obtidos nesta área em estudo foi 957.8, 132 e 301metros, os quais indicam a distância máxima de valores correlacionados entre si nas suas respectivas profundidades, por outro lado este parâmetro também indica a distância máxima que as amostras podem ser coletadas antes de apresentar comportamento aleatório, como conveniente na estatística clássica (MEDEIROS, 2015).

A dependência espacial foi caracterizada de duas formas de acordo com os métodos descritos por Cambardella (1994) e Seidel \& Oliveira (2014), ilustrado na Tabela 2 como, GDE e IDE respectivamente. A dependência forte de características do solo, demonstram que a variabilidade é controlada por fatores intrínsecos, como textura e mineralogia, já as variáveis que apresentam dependência espacial fraca tem suas variáveis controladas, ou seja, com o preparo do solo (VIEIRA \& COL, 1983).

A dependência obtida pelo primeiro método (GDE), resultou em 20,83\%, na camada superior $(1 \mathrm{~cm})$, indicando alta correção das amostras, ou seja, menor que $25 \%$, conforme classificada anteriormente e 44,07 e 49,72\% nas camadas 10 e $20 \mathrm{~cm}$ respectivamente, indicando moderada correlação de amostras, dentro do índice entre 25 e $75 \%$.

O segundo método de mensuração de dependência espacial (IDE), o qual deve ser considerado nos parâmetros do modelo de ajuste esférico, percentuais entre 0 e $31,7 \%$, o qual resultou na camada superficial de $1 \mathrm{~cm} \mathrm{29,62 \%} \mathrm{de} \mathrm{dependência}$ espacial, 4,95 e $9,18 \%$ para 10 e $20 \mathrm{~cm}$ respectivamente, sendo que quanto mais próximo de $0 \%$ menor dependência espacial.

Geralmente fenômenos observados na natureza, não apresentam dependência espacial de $100 \%$, os seus índices estão diretamente ligados ao modelo de ajuste do semivariograma, que explica a dependência espacial das amostras (SEIDEL \& OLIVEIRA, 2014). Os dois índices encontrados tiveram resultados distintos, pois o primeiro método considera somente efeito pepita e patamar, já o segundo método considera fator do modelo, alcance pratico, distância máxima entre amostras, efeito pepita e patamar. Podendo-se considerar que este último inclui maior confiabilidade dos resultados dos índices, já que não considera as variáveis de maneira generalizada, considerando, portanto, fatores do semivariograma e correlograma. 
Com isto, pôde-se compreender a influência do manejo recente no solo da área estudada, pois houve maior homogeneidade de amostras, nas profundidades inferiores a $25 \mathrm{~cm}$ de profundidade, sendo este o perfil que foi realizado de descompactação do solo, com isto, entende-se que o manejo influenciou diretamente na análise, visto que, foi nestas que houve grau de dependência forte $\mathrm{e}$ moderada.

\section{Mapeamento da umidade do solo e RSP}

Com base nas análises de estatística espacial (geoestatística), foi possível escolher o método de interpolação dos dados, sendo que onde houve dependência espacial, foi feito interpolação pelo método krigagem ordinária, sendo eles nas profundidades de 1,10 e $20 \mathrm{~cm}$, já para os dados que não obtiveram dependência espacial, utilizou-se interpolação pelo método inverso da distância ponderada (IDW), sendo eles: umidade do solo e nos perfis de $30,40 \mathrm{~cm}$.

A Figura 4 é a representação gráfica dos percentuais da umidade do solo, os quais estão entre $3,11 \%$ e 9,50 \%, estes teores são representados por variações da cor azul, onde quanto mais forte o tom, maior índice de umidade, nota-se, portanto, que o maior teor encontra-se na parte superior da área e menor na parte de baixo, com algumas manchas ao longo da área.

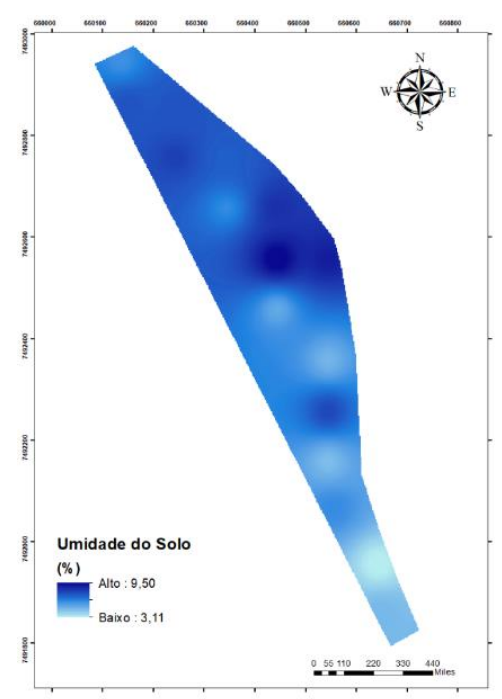

Figura 4: Mapa do percentual da umidade do solo da área da Fazenda Santa Martha.

Os índices da RSP, foram representados através de mapas em três intervalos de cores diferentes, sendo elas: vermelha, amarela e verde, representando teores de compactação como alto, médio e baixo, respectivamente, os parâmetros foram fixados para cada profundidade, ou seja, estes teores foram flexíveis conforme a profundidade de cada perfil.

Os índices de compactação de todos os perfis estão ilustrados na Figura 5, onde a Figura 5a, representa compactação no perfil de $1 \mathrm{~cm}$, com variação da RSP entre 0,09 a $0,51 \mathrm{Mpa}$, na Figura 5b (perfil 10 $\mathrm{cm}$ ) foi entre 0,24 e 1,29 Mpa, na Figura $5 \mathrm{c}$ (perfil $20 \mathrm{~cm}$ ) entre 1,08 e 2,19 $\mathrm{MPa}$, na Figura 5d (perfil $30 \mathrm{~cm}$ ) entre 1,13 e 4,48
Mpa e na Figura 5e (perfil $40 \mathrm{~cm}$ ) entre 1,40 e 4,78 $\mathrm{MPa}$. Os índices de compactação do solo desta área, comportase de maneira crescente, conforme aumenta a profundidade, aumenta a RSP, com menor índice no perfil mais superficial $(1 \mathrm{~cm})$ e maior no mais profundo $(40 \mathrm{~cm})$, é possível notar ainda que a RSP mais intensa está na parte superior da área (manchas vermelhas), nas profundidades de 1 a $20 \mathrm{~cm}$, e uma distribuição mais homogênea nas demais profundidades, as mais profundas (30 e 40 $\mathrm{cm})$. 


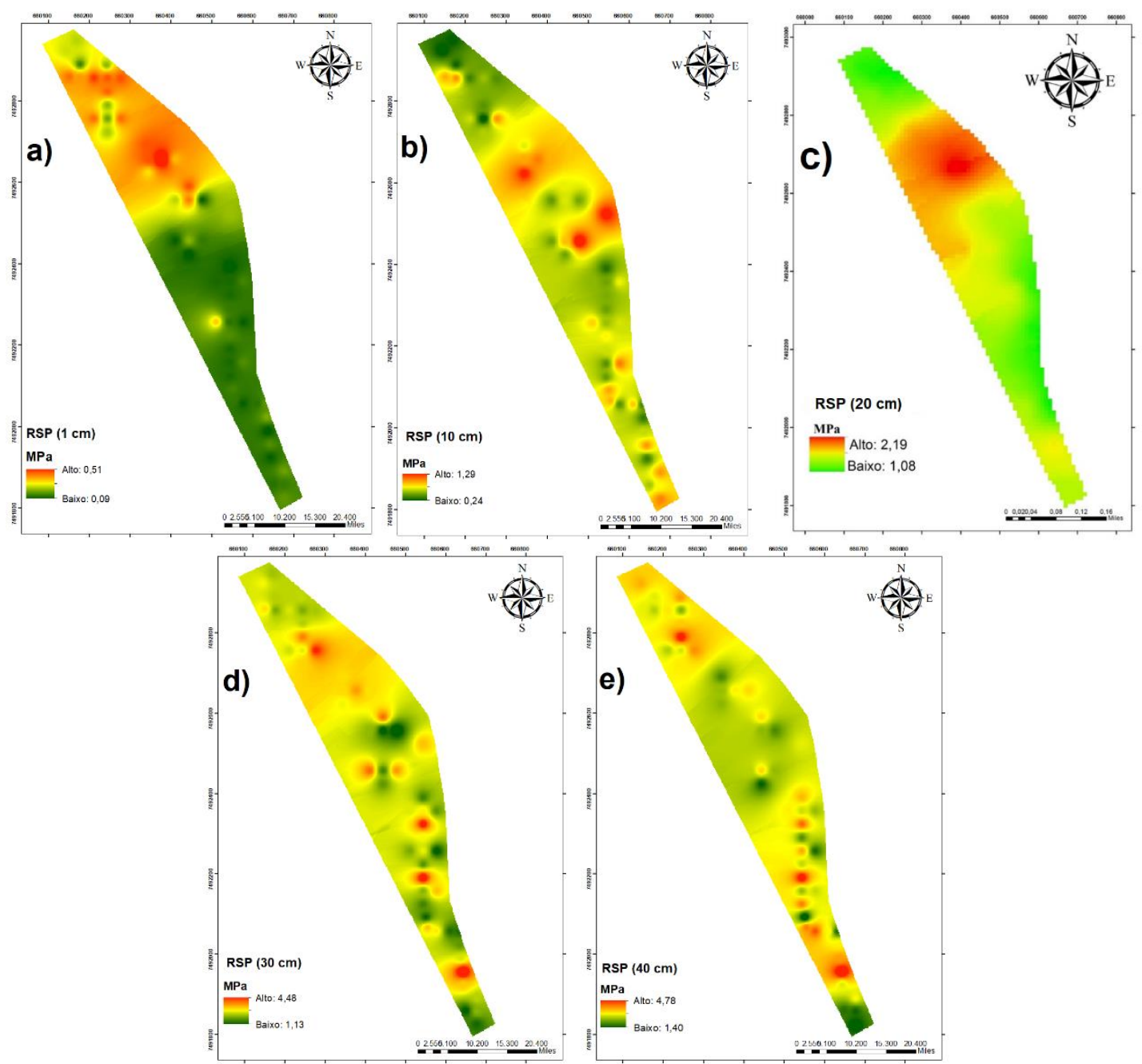

Figura 5: Mapas de resistência do solo à penetração nas profundidades 1, 10, 20, 30 e $40 \mathrm{~cm}$ da área da Fazenda Santa Martha.

De acordo com as ilustrações do mapa de umidade do solo e os de RSP, nota-se que há correlação entre a intensidade de compactação e umidade, principalmente nas análises feitas nos perfis igual ou inferior a $20 \mathrm{~cm}$, em que nos locais com maior índice de água no solo, mais compactado o local. Resultados semelhantes foram encontrado por Medeiros (2015), em que quanto maior quantidade de água no solo maior a compactação, já Hillel (1998), encontrou o inverso, quanto menor disponibilidade de

\section{CONCLUSÕES}

O modelo de semivariograma que melhor se ajustou aos valores de resistência do solo à penetração foi 0 esférico. água no solo maior compactação. Isto pode estar diretamente ligado ao tipo de solo analisado e manejo do mesmo.

As análises espaciais representando o comportamento do solo são importantes no planejamento de ações de manejo, devido ao fato da importância de estimativas de locais não amostrados. De acordo com Cambardella (1994), conhecer e entender a distribuição das propriedades do solo é importante para aplicar técnicas de manejo de maneira localizada e também para determinar efeitos no ambiente.
Foi possível determinar a distribuição da RSP por meio de método geoestistístico, alcançando boa 
dependência espacial em perfis de profundidade do solo em 1,10 e $20 \mathrm{~cm}$.

A umidade do solo influenciou a RSP nas camadas superficiais.

\section{REFERÊNCIAS BIBLIOGRÁFICAS}

Asae. American Society of Agricultural Engineering. Spray nozzle classification by droplet spectra. St. Joseph: ASAE (ASAE Standard S572 FEB04). p.411-14. 2004.

Bacchi, O.O.S. Efeitos da compactação sobre o sistema solo-planta em cultura de cana-de-açúcar. 1976. $67 \mathrm{f}$. Dissertação (Mestrado em agronomia). Escola Superior de Agronomia Luiz Queiroz, Piracicaba -SP,67 p. 1976.

Brito, L. F.; Marques, J.; Pereira, G. T.; La Scala, N. Spatial variability of soil $\mathrm{CO}_{2}$ emission in different topographic positions. Bragantia, v.69, p.19-27, 2010. http://dx.doi.org/10.1590/S0006-

87052010000500004.

Brocca, L.; Morbidelli, R.; Melone, F.; Moramarco, T. Soil moisture spatial variability in experimental areas of central Italy. Journal of Hydrology, v.333, p.356373, 2007. https://doi.org/10.1016/j.jhydrol.2006.09.0 04.

Cambardella, C. A.; Moorman, T. B.; Novak, J. M.; Parkin, T. B.; Karlen, D. L.; Turco, R. F.; Konopka, A. E. Fieldscale variability of soil properties in central Iowa soils. Soil Science Society of America Journal Abstract, v.58, p.15011511 , 1994.

Doi:10.2136/sssaj1994.036159950058000 50033x.

Cepagri - Centro de Pesquisas Meteorológicas e Climáticas Aplicadas à Agricultura, Clima dos Municípios Paulista. Disponível em> http://www.cpa.unicamp.br. 2017.

Colet, M. J.; Sverzut, C. B.; Weirich Neto, P. H.; Souza, Z. M. Alterações em atributos físicos de um solo sob pastagem após escarificação. Ciência Agrotecnologica, v. 33, n. 2, p. 361-368, 2009. http://dx.doi.org/10.1590/S141370542009000200001 .
A descompactação do solo no perfil de $25 \mathrm{~cm}$, influenciou de forma positiva na homogeneidade das amostras submetidas a análise.

Curi, N.; Larach, J. O. I.; Kämpf, N.; Moniz, A.C. ; Fontes, L.E.F. Vocabulário da ciência do solo. Campinas: Sociedade Brasileira de Ciência do Solo, 90 p. 1993. Embrapa. Empresa Brasileira de Pesquisa Agropecuária. Centro Nacional e Pesquisa em Solos. Sistema brasileiro de classificação de solos. Brasília, 306 p. 2006.

Embrapa. Empresa Brasileira De Pesquisa Agropecuária. Ministério da Agricultura, pecuária e abastecimento. Compactação do solo: Consequências para o crescimento vegetal. 2016.

Hilleil, D. Environmental soil plysics . San Diego: Academic Press, 1998. 771p.

Lima, R. P.; De León, M. J.; Da Silva, A. R. Compactação do solo de diferentes classes texturais em áreas de produção de cana-de-açúcar - Revista Ceres, Viçosa, v. 60, n.1, p. 016-020, jan/fev, 2013. http://dx.doi.org/10.1590/S0034-

737X2013000100003.

Medeiros, J. L. F. Variabilidade espacial da resistência à penetração e do teor de água em solo cultivado com cana-deaçúcar no litoral sul potiguar. 51 f. 2015. Dissertação. Mestrado em Manejo de Solo e Água. Universidade Federal Rural do Semi-Árido (UFERSA). Mossoró-RN. 2015.

Molin, J. P., Dias, C.T.S.; Carbonera, L. Estudos com penetrometria: Novos equipamentos e amostragem correta. Revista Brasileira de Engenharia Agrícola e Ambiental, Campina Grande, v.16, n.5, p.584-590. 2012.

Ohashi, M.; Gyokusen, B. Temporal change in spatial variability of soil respiration on a slope of Japanese cedar (D. Don) forest. Soil Biology Biochemistry, v.39, p.1130-1138, 2007. https://doi.org/10.1016/j.soilbio.2006.12.0 21. 
Oliveira, I. A.; Campos, M. C. C.; Soares, M. D. R.; Aquino, R. E.; Marques Júnior, J.; Nascimento, E. P. Variabilidade espacial de atributos físicos em um cambissoloháplico, sob diferentes usos na região sul do Amazonas. Revista Brasileira de Ciência do Solo, Viçosa, MG,v. 37, n. 1, p. 1103-1112, 2013. http://dx.doi.org/10.1590/S010006832013000400027.

Oliveira, M. S., Seidel, E. J. Novo índice geoestatístico para a mensuração da Dependência espacial. Revista Brasileira de Ciências do Solo. v. 38, p. 699-705, 2014.

Panosso, A. R.; Marques, J.; Pereira, G. T.; La Scala, N. Spatial and temporal variability of soil $\mathrm{CO} 2$ emission in a sugarcane area under green and slash-andburn managements. Soil Tillage Research, v.105, p.275-282, 2009. https://doi.org/10.1016/j.still.2009.09.008.

Ralisch, R.; Miranda, T.M.; Okomura, R.S.; Barbosa, G.M.C.; Guimarães, M. F.; Scopel, E. ; Balbino, L.C. Resistência à penetração de um Latossolo Vermelho.

Revista Brasileira de Engenharia Agrícola e Ambiental. v.12, n.4, p.381384, 2008.

Robertson, G. P. 2004. GS+: Geoestatistícs for the environmental sciences - GS+ Use(s Guide. Plainwell, Gamma Desing Software, p.152, 2004.

Roque, M. W.; Matsura, E. E.; Souza, Z. M.; Bizari, D. R.; Souza, A. L. Correlação linear e espacial entre a resistência do solo ao penetrômetro e a produtividade do feijoeiro irrigado. Revista Brasileira de Ciência do Solo, v. 32, p. 1827-1835, 2008. http://dx.doi.org/10.1590/S010006832008000500004.

Roque, A. A. O.; Souza, Z. M.; Barbosa, R. S.; Souza, G. S. Controle de tráfego agrícola e atributos físicos do solo em área cultivada com cana-de-açúcar. Pesquisa
Agropecuária Brasileira, Brasília, v.45, n.7, p.744-750, 2010.

Silva, V. R. et al. Variabilidade espacial da resistência do solo à penetração em plantio direto. Ciência Rural, Santa Maria,v.34, n.2, p.399-406, 2004.

Souza, Z.M. et al. Dependência espacial da resistência do solo à penetração e teor de água do solo sob cultivo de cana-deaçúcar. Ciência Rural, Santa Maria, v.36, n.1, p.128-134, 2006. http://dx.doi.org/10.1590/S0103-

84782006000100019.

Vieira, S.R; Lombardi Neto, F. Variabilidade espacial do potencial de erosão das chuvas do Estado de São Paulo. Bragantia, v.54, n.2, p.405-412. 1995. http://dx.doi.org/10.1590/S000687051995000200019.

Vieira, S.R. Geoestatística em estudos de variabilidade espacial do solo. In: NOVAIS, R.F. et al. (Eds). Tópicos em ciência do solo. Viçosa: Sociedade Brasileira de Ciência do Solo, v.1, p.1-53, 2000.

Vieira, S.R; Hatfield, J.L.; Nielsen, D.R; Biggar, JW. Geostatistical theory and application to variability of some agronomical properties. Hilgardia, v.51, n.3, p.1-75. 1983. DOI:10.3733/hilg.v51n03p075.

Zimback, C. R. L. Análise espacial de atributos químicos de solos para fins de mapeamento da fertilidade do solo. 2001. 114 f. Tese (Livre-Docência)Faculdade de Ciências Agronômicas, Universidade Estadual Paulista, Botucatu, 2001.

Zhu, Y.; Shao, M. Variability and pattern of surface moisture on a small-scale hillslope in Liudaogou catchment on the northern Loess Plateau of China. Geoderma, v.147, p.185-191, 2008. https://doi.org/10.1016/j.geoderma.2008.08 .012 . 